\title{
A MEAN VALUE THEOREM FOR THE HEAT EQUATION
}

W. FULKS

The Gauss mean value theorem and its converse, due to Koebe, characterize solutions of Laplace's equation [2]. In view of the strong analogy between Laplace's equation and the heat equation it seems reasonable to expect an analogue of the Gauss-Koebe result to hold for the heat equation. The purpose of this paper is to present such a result. For simplicity we work in two dimensions, though it is clear from the calculations that the result is independent of dimensions. Murakami [3] has used a limit form of the mean value formula we establish here on which to base his discussion of generalized parabolic operators.

Let $H$ and $H^{\prime}$ be the heat operator and its adjoint respectively:

$$
H u=u_{x x}-u_{t}, \quad H^{\prime} u=u_{x x}+u_{t} .
$$

Then

$$
u H^{\prime} v-v H u=\left(u u_{x}-v v_{x}\right)_{x}+(u v)_{t} .
$$

By the divergence theorem, using $(\xi, \tau)$ as integration variables, we get

$$
\int_{D} \int\left[u H^{\prime} v-v H u\right] d \xi d \tau=\int_{B}\left[\left(u v_{\xi}-v u_{\xi}\right) n_{1}+u v n_{2}\right] d s
$$

where $n=\left(n_{1}, n_{2}\right)$ is the exterior unit normal to $B=\partial D$. We assume of course sufficient smoothness to apply the divergence theorem. If $H u=H^{\prime} v=0$, then (2) becomes

$$
\int_{B}\left[\left(u v_{\xi}-v u_{\xi}\right) n_{1}+u v n_{2}\right] d s=0
$$

and if $v \equiv 1$ this reduces to

$$
\int_{B}\left[-u_{\xi} n_{1}+u n_{2}\right] d s=0
$$

As was pointed out by Goursat [1], this last result is analogous to the formula $\int_{B}(\partial u / \partial n) d s=0$ for harmonic function. The following result, essentially given in [1], points up this analogy.

TheOREM 1. Let $R$ be an open region and let $u$ be a solution of $H u=0$

Received by the editors November 30, 1964. 
in $R$. Then (4) holds for every contour (simple closed rectifiable curve) which together with its interior lies in $R$. Conversely, if $u$ and $u_{x}$ are continuous in $R$, and (4) holds for every such contour, then $H u=0$ in $R$.

Proof. The first part of the theorem has been established by the calculations leading up to (4). To prove the second part observe that (4) can be written in the form

$$
\int_{B} u d \xi+u_{\xi} d \tau=0
$$

so that the integral is independent of the path in $R$. Hence $\left(u, u_{\xi}\right)$ is the gradient of a function $v$ in $R$. We have then

so that

$$
v_{\xi}=u, \quad v_{\tau}=u_{\xi},
$$

$$
v_{\xi \xi}=u_{\xi}=v_{\tau} .
$$

Thus $v$ is a solution of the heat equation in $R$, and hence $v \in C^{\infty}$, so that in $R$,

$$
H u=H v_{\xi}=(H v)_{\xi}=0 .
$$

The fundamental solution $k$ of the heat equation is given by

$$
k(x, t)= \begin{cases}(4 \pi t)^{-1 / 2} \exp \left(x^{2} / 4 t\right), & t>0 \\ 0, & t \leqq 0 .\end{cases}
$$

The curve described by

$$
k\left(x_{0}-\xi, t_{0}-\tau\right)=c
$$

where $\left(x_{0}, t_{0}\right)$ is a fixed point and $c$ a positive constant, is a smooth convex curve lying in the half plane $\left\{\tau \leqq t_{0}\right\}$ and symmetric about the line $\left\{\xi=x_{0}\right\}$. As $c \rightarrow \infty$ it shrinks to the point $\left(x_{0}, t_{0}\right)$. We denote this curve by $B\left(x_{0}, t_{0} ; c\right)$ and usually delete the arguments. By $B_{t} \equiv B_{t}\left(x_{0}, t_{0} ; c\right)$ we mean $B \cap\{\tau \leqq t\}$. We further define $D \equiv D\left(x_{0}, t_{0}, c\right)$ as the interior of $B, D_{t} \equiv D \cap\{\tau \leqq t\}$ and $I_{t} \equiv D \cap\{\tau=t\}$, and will be concerned only with the case where $t_{1} \leqq t \leqq t_{0}, t_{1}$ being the minimum value of $\tau$ on $B$.

If $u$ is a solution of the heat equation in an open region $R$, and $\left(x_{0}, t_{0}\right)$ is a point in $R$, then $B$ (and hence $D$ ) will lie in $R$ for $c$ sufficiently large, say $c \geqq c_{0}$. Denoting $k\left(x_{0}-\xi, t_{0}-\tau\right)$ simply by $k$ we apply (3) with $v=k$ to $\partial D_{t}=B_{t} \cup I_{t}$. Thus

$$
\int_{B_{t} \cup I_{t}}\left[\left(u k_{\xi}-k u_{\xi}\right) n_{1}+u k n_{2}\right] d s=0 .
$$


On $I_{t}$ we have $n_{1}=0, n_{2}=1$ so that

$$
-\int_{I_{t}} u k d x=\int_{B_{t}} u k_{\xi} n_{1} d s+\int_{B_{t}} k\left[-u_{\xi} n_{1}+u n_{2}\right] d s
$$

or

$$
\int_{I_{t}} u k d x=\int_{B_{t}} u\left(-k_{\xi} n_{1}\right) d s-c \int_{B_{t}}\left[-u_{\xi} n+u n_{2}\right] d s,
$$

since $k=c$ on $B$ and hence on $B_{t}$. (We note that (3) is an undirected line integral so that the signs in the two last equations are correct.)

If we let $t \rightarrow t_{0}-0$ we get, formally,

$$
\begin{aligned}
& u\left(x_{0}, t_{0}\right)=\int_{B} u\left(-k_{\xi} n_{1}\right) d s-c \int_{B}\left[-u_{\xi} n_{1}+u n_{2}\right] d s, \\
& u\left(x_{0}, t_{0}\right)=\int_{B} u\left(-k_{\xi} n_{1}\right) d s,
\end{aligned}
$$

using equation (4) and the $\delta$-function property of $k$ at $t=t_{0}$. We proceed to examine the limiting procedure more carefully to justify the deduction of (6) from (5).

One easily computes $n_{1}$ and $k_{\xi}$ on $B$ to get

$-k_{\xi} n_{1}=c\left(x_{0}-\xi\right)^{2}\left\{4\left(x_{0}-\xi\right)^{2}\left(t_{0}-\tau\right)^{2}+\left[2\left(t_{0}-\tau\right)-\left(x_{0}-\xi\right)^{2}\right]^{2}\right\}^{-1 / 2}$.

If we set

$$
Q(x, t)=c x^{2}\left[4 x^{2} t^{2}+\left(2 t-x^{2}\right)^{2}\right]^{-1 / 2},
$$

then (6) becomes

$$
u\left(x_{0}, t_{0}\right)=\int_{B} Q\left(x_{0}-\xi, t_{0}-\tau\right) u(\xi, \tau) d s .
$$

We first examine $Q$. Setting $x_{0}-\xi=x, t_{0}-\tau=t$ we estimate $Q$ for $x, t$ near $(0,0)$ for $c$ fixed. On $B, x^{2}=-2 t \log \left(4 \pi c^{2} t\right)$, so that $Q$ becomes, as $t \rightarrow 0$

$$
\begin{aligned}
& \frac{-c 2 t \log \left(4 \pi c^{2} t\right)}{\left[-8 t^{3} \log \left(4 \pi c^{2} t\right)+\left[2 t+2 t \log 4 \pi c^{2} t\right]^{2}\right]^{1 / 2}} \\
& =\frac{-c 2 t \log \left(4 \pi c^{2} t\right)}{\left(\left(2 t \log 4 \pi c^{2} t\right)^{2}[1+O(1)]\right)^{1 / 2}} \sim c .
\end{aligned}
$$

Thus $Q$ is bounded and continuous on $B$ and the first term on the right side of (5) has the limit indicated, namely the right side of (6). 
The second term on the right side of (5) gives no trouble, so we turn to left side of (5). This is

$$
\int_{I_{t}} k\left(x_{0}-\xi, t_{0}-t\right) u(\xi, t) d \xi
$$

By the continuity of $u$ at $\left(x_{0}, t_{0}\right)$ we have $u(\xi, t)=u\left(x_{0}, t_{0}\right)+\eta$, where $\eta \rightarrow 0$ as $(\xi, t) \rightarrow\left(x_{0}, t_{0}\right)$; in particular sup I $_{t}|\eta| \rightarrow 0$ as $t \rightarrow t_{0}$. Thus

$$
\int_{I_{t}} k u d \xi=u\left(x_{0}, t_{0}\right) \int_{I_{t}} k\left(x_{0}-\xi, t_{0}-t\right) d \xi+O(1) \text { as } t \rightarrow t_{0}
$$

And

$$
\int_{I_{t}} k\left(x_{0}-\xi, t_{0}-\tau\right) d \tau=\int_{-x_{1}}^{x_{1}} k(x, t) d t
$$

where $x_{1}=\left(-2 t \log \left(4 \pi c^{2} t\right)\right)^{1 / 2}$. Substituting $x^{2} / 4 t=y$ we get

$$
\frac{1}{\sqrt{ } \pi} \int_{-x_{1} / 2 \sqrt{ } t}^{-x_{1} / 2 \sqrt{ } t} \exp \left(-y^{2}\right) d y \rightarrow \frac{1}{\sqrt{ } \pi} \int_{-\infty}^{\infty} \exp \left(-y^{2}\right) d y=1 \text { as } t \rightarrow 0 .
$$

This justifies (6) and (8) and establishes the following.

THEOREM 2. Let $u$ be a solution of the heat equation in an open region $R$. Then for any $(x, t) \in R$, there is a $c_{0}>0$ such that for $c \geqq c_{0}$

$$
u(x, t)=\int_{B} Q(x-\xi, t-\tau) u(\xi, \tau) d s
$$

where $B=B(x, t ; c)$ and $Q$ is defined by $(7)$.

We note the following:

$$
Q(x, t)>0, \quad \text { on } B
$$

and by setting $u \equiv 1$ in (8) we get

$$
\int_{B} Q(x-\xi, t-\tau) d s \equiv 1 \text {. }
$$

It is also easy to verify that for any fixed $(x, t)$ and $c$, the curve $B(x, t ; c)$ has a horizontal tangent at the point $(x, t)$.

We proceed to build up to the analogue of Koebe's theorem, through the maximum principle.

If $R$ is an open region and $P_{0}=\left(x_{0}, t_{0}\right)$ is a point in $R$, then we will denote by $R_{P_{0}}$ the set of points $(\xi, \tau) \in R$ which can be connected to $\left(x_{0}, t_{0}\right)$ by a polygonal line on which $t$ is strictly increasing as the line 
is described from $(\xi, \tau)$ to $\left(x_{0}, t_{0}\right) . R_{P_{0}}$ is called the influence region of $P_{0}$ relative to $R$. Let $u$ be a continuous function in $R$. If the assumption of a maximum or minimum by $u$ at a point $P \in R$ implies $u \equiv$ const in $R_{P}$ we say that $u$ satisfies the parabolic maximum principle. It is well known [4] that solutions of the heat equation satisfy this principle.

Further, if $P_{0}=\left(x_{0}, t_{0}\right) \in R$, then there is a $c_{0}$ such that $B\left(x_{0}, t_{0} ; c\right) \subset R$ for all $c \geqq c_{0}$. If $u$ is a continuous function with the property that for $(x, t) \in R$

$$
u(x, t)=\int_{B} Q(x-\xi, t-\tau) u(\xi, \tau) d s
$$

for all $c \geqq c_{0}$ (where $c_{0}$ is determined as above) then we will say that $u$ satisfies the parabolic mean value property in $R$. We relate these concepts in the following theorem.

THEOREм 3. Let $u$ be a continuous function in the open region $R$, and let $u$ satisfy the parabolic mean value property there. Then $u$ satisfies the parabolic maximum principle in $R$.

Proof. Suppose $u$ attains a maximum (or a minimum) at a point $P_{0}=\left(x_{0}, t_{0}\right) \in R$, and let $P_{1}=\left(x_{1}, t_{1}\right) \in R_{P_{0}}$. There is then a polygonal line $L$ in $R_{P_{0}}$ connecting $P_{1}$ to $P_{0}$ on which $t$ is monotone increasing. Since $L$ is bounded away from the boundary of $R_{1}$ there is a $c_{0}$ such that $B(x, t ; c) \subset R$ for every $(x, t)$ on $L$ and every $c \geqq c_{0}$. It follows from (9) and (10) that $u(x, t) \equiv u\left(x_{0}, t_{0}\right)$ in the set swept out by $B\left(x_{0}, t_{0} ; c\right)$ for $c \geqq c_{0}$, and hence that $u \equiv u\left(x_{0}, t_{0}\right)$ on a segment of $L$ ending at $\left(x_{0}, t_{0}\right)$. One then iterates this argument proceeding stepwise down $L$. Since $L$ has a minimum slope one arrives at $P_{1}$ after finite number of steps and concludes that $u\left(x_{1}, t_{1}\right)=u\left(x_{0}, t_{0}\right)$.

We can now deduce the analogue of Koebe's theorem.

THEOREM 4. Let $u$ be a continuous function which satisfies the parabolic mean value property in an open region $R$. Then $u$ is a solution of the heat equation in $R$.

Proof. Let $\left(x_{0}, t_{0}\right) \in R$. Then there is a closed rectangle, $R^{\prime}$, centered at $\left(x_{0}, t_{0}\right)$ with sides parallel to the axes lying in $R$. Let $v$ be the solution of the heat equation in $R^{\prime}$ which achieves the values $u$ on the "lower boundary" of $R^{\prime}$ consisting of the bottom and sides of $R^{\prime}$. This $v$ can be expressed as a "Poisson integral" in terms of the Green's function for $R^{\prime}$. In the interior of $R^{\prime}$, both $u$ and $v$ satisfy the parabolic mean value property, hence so does their difference. Thus the difference satisfies also the maximum principle and must therefore 
achieve both its maximum and minimum on the lower boundary. The difference vanishes there, so that $u \equiv v$ in $R^{\prime}$. Thus $u$ is a solution of the heat equation at $\left(x_{0}, t_{0}\right)$, and since $\left(x_{0}, t_{0}\right)$ is arbitrary in $R, u$ is a solution of the heat equation throughout $R$.

In the case of harmonic functions one gets as a trivial consequence of the Gauss theorem that a similar result holds for means over the interior of circles (or spheres in higher dimensions). Whether or not an analogous result holds for the heat equation is not clear. In any case, it is not as immediate as in the harmonic case.

\section{BIBLIOGRAPHY}

1. E. Goursat, Cours d'analyse, Tome III, Gauthier-Villars, Paris, 1942.

2. O. D. Kellogg, Foundations of potential theory, Dover, New York.

3. H. Murakami, Semi-linear partial differential equations of parabolic type, Funkcial. Ekvac. 3 (1960), 1-50.

4. L. Nirenberg, $A$ strong maximum principle for parabolic equations, Comm. Pure Appl. Math. 6 (1953), 167-177.

University of Colorado 